\title{
Repetitive Transcranial Magnetic Stimulation Treatment Adherence and the Research Progress of Nursing Intervention in the Patients with Mental Disorders
}

\author{
Shao Chen ${ }^{1 \dagger}$, Juan Zhang ${ }^{1 \dagger}$, Yunshu Zhang ${ }^{2}$ and Keqing $\mathbf{L i}^{\mathbf{2}^{*}}$ \\ ${ }^{1}$ Nursing College of Hebei University, Baoding, Hebei 071000, China \\ ${ }^{2}$ Sixth People's Hospital of Hebei Province, Baoding, Hebei 071000, China
}

\begin{abstract}
By reviewing literatures, the author summarized the influence factors of compliance in rTMS treatment. The major factors were found to be involved patient factors, medical factors, associated-therapeutic factors and social supporting factors and put forward corresponding nursing intervention measures, in order to improve the patients' compliance, satisfaction and the treatment effect.
\end{abstract}

\author{
KEYWORDS \\ rTMS \\ Compliance \\ Mental disorders \\ Nursing
}

\section{Introduction}

Repetitive Transcranial Magnetic Stimulation (rTMS) is a kind of magnetic stimulation, which acts on the central nervous system by pulsed magnetic field, regulates the action potential of nerve cells through induced current, thus affects the neurophysiological activity [1]. The technology has many advantages, such as ease of operation, noninvasive, safe and reliable [2], and it has been widely used in neurology, psychiatry and other fields [3,4]. However, some investigation showed that the treatment compliance of rTMS treatment compliance in the patients with mental disorders in China was low [5,6]. By reviewing the literatures, this paper concluded the rTMS treatment compliance affecting factors and nursing interventions, so as to provide reference for nursing care for such patients.

\section{Compliance status of patients with mental disorders in}

Copyright ( $(2016$ Shao Chen et al.

doi: 10.18686/jn.v5i1.4

This is an open-access article distributed under the terms of the Creative Commons Attribution Unported License (http://creativecommons.org/ licenses/by-nc/4.0/), which permits unrestricted use, distribution, and reproduction in any medium, provided the original work is properly cited.

${ }^{\star}$ Corresponding author: Sixth People's Hospital of Hebei Province, Baoding, Hebei 071000, China. E-mail: like1002@sina.com

${ }^{\dagger}$ These authors contributed equally to this manuscript.

\section{rTMS treatment}

Kaiwen Wu [5] did rTMS treatment compliance questionnaire survey which included 108 patients with mental disorders. The results show that only $10(9.26 \%)$ patients were fully compliance, partial compliance and noncompliance of patients accounted for $90.74 \%, 94.44 \%$ of patients have varying degrees of anxiety, the rTMS treatment adherence of most patients was low. Min Liu [6] investigated 93 cases of mental disorders, which was similar to $\mathrm{Wu}$ Kaiwen's results, it showed that only nine cases (9.8\%) patients were fully compliance, $86(92.5 \%)$ patients haved varying degrees of anxiety, more than half of the patients haved obvious anxiety. The results showed that the treatment compliance of rTMS in patients with mental disorders was poor.

\section{The influence factors of rTMS treatment adherence}

\subsection{Patient factors}

Due to the particularity of mental illness, the patients with mental disorder are prone to low self-esteem, anxiety, depression. They not only bear the pain of the disease, also suffer great psychological pressure, and have serious stigma [7]. Research shows that is the reason of large gap between the prevalence rate and treatment rate of mental disorders 
[8]. The side effects of long-term drug treatment and recurrent disease lead to a decline in the patient's psychological endurance. They lose confidence in the treatment and life, All of these reasons lead to a low rTMS treatment's enthusiasm. Some patients are dominated by the psychiatric symptoms, such as persecutory delusion and hallucinations, They are serious lack of trust, and therefore refuse treatment. Some involuntary inpatients lack of proper understanding, leading to poor compliance. Some patients with hypersensitivity have pain perception beyond the ordinary. If they do not understand the treatment process, they may fear during the treatment of rTMS, fearing the sequelae, so as not to receive treatment.

\subsection{Medical factors}

Sometimes, doctors and nurses because of busy work do not make a patient explained for patients or ignore the patient's health education and psychological care. However, the patients acquire the knowledge of treatment mainly from the medical knowledge. Less communication between nurses and patients lead to patients lack of the understanding of the disease and treatment. All of these lead to poor compliance.

\subsection{Aassociated-therapeutic factors}

RTMS is considered to be relatively safe. A large number of clinical trials and meta analysis conclusions also support the treatment applied in clinical [9]. But there still are some side effects in the process of rTMS treatment. It may cause patient discomfort, such as head and neck discomfort, hearing impairment, and so on [10,11]. Some patients are difficult to tolerate the discomfort, so unwilling to accept this treatment. In addition, if the treatment environment is very noisy, it may cause that the patients are irritable, unable to complete treatment. Further more, rTMS treatment itself also can produce noise in the process of treatment, some patients can't stand the noise, especially in the process of treatment of elderly patients.

\section{3. $\square$. Social supporting factors}

Social supporting is an important factor for the patient compliance, and it is proportional to the health-promoting behaviors [12]. Patients' family support is the main source of social supporting. Family members as the primary caregivers of patients' living, play an important role in the patient's treatment and rehabilitation [13]. Some patients have not a good family support during hospitalization, such as rarely be visited by the family, so they may lose confidence in life and not active in treatment. Some patients have a heavy burden of disease, fearing of the cost of treatment or the treatment effect less than expected, the patient may refuse the treatment, especially those with severe mental disorders[14,15].

\section{Nursing intervention of the rTMS treatment}

\subsection{The relationship between nurses and patients}

Establishing a good relationship with the patient is an important foundation to improve treatment compliance. Doctors and nurses should strengthen the communication with patients, answer to questions and doubts more patiently, and provide more help and information for patients and their families. Various measures can be taken to establish a good nurse-patient relationship. 60 cases of patients with depression were randomly divided into two groups by Xiuchun Gao, all the patients were treated by rTMS [16]. The control group was taken care of routine nursing care, the intervention group received psychological nursing intervention on the basis of routine nursing care. The authors compared two groups before and after the intervention by using the Hamilton Depression Scale (HAMD). The results showed that the intervention group scored lower than the control group, the efficacy and treatment compliance in the intervention group were better than the control group. We can conclude that psychological intervention can improve rTMS treatment compliance, and improve the patient's mental capacity and the quality of nursing. 63 cases of anxiety patients were divided into experimental group and control group, all of them received the treatment of rTMS and drugs [17]. In addition, only the experimental group received psychological care. The results showed that combination therapy can effectively reduce the onset time of rTMS treatment. It can indirectly improve the patients' compliance. Wei Zheng also found that the effect of rTMS treatment with psychological care was better than the simple rTMS treatment. Compliance can be improved by doing so [18].

\subsection{Health education}

RTMS is a relatively new way of treatment, compared with the previous drug therapy. Many patients are not familiar with the treatment process, enhancing the health education in them is particularly important. Health education content should include the purpose of rTMS treatment, principle, implementation process and matters needing attention, common adverse reactions, etc. The nurse should strengthen the study of mental disorders and rTMS related knowledge, improve professional ability and the patients' trust. 101 cases of mental disorders were randomly divided into observation group and control group by Qingchun Ai [19]. All the patients were received treatment of rTMS. Two groups were accepted routine nursing care. In addition, the observation group were interviewed twice a week. The result was the observation group to treatment completion was significantly better than the control group. The object of health education should also include patients' families. Bernard [20] suggested that the family should watch the video of rTMS together with the patient. Some knowledge of materials including video, manuals, related website information can be provided by nurse. The results indicated that these measures could enable patients and their families better understand the relevant knowledge of rTMS, help to improve treatment compliance. 


\subsection{The side effects of rTMS}

The characteristic of mental disorders are lack of insight. Therefore, corresponding nursing measures are necessary for them. A study about rTMS treatment suggested that creating a comfortable treatment environment, providing a headset and playing soft music for patients when they have the treatment of rTMS can obviously reduce the incidence of adverse reactions [21]. Another study also pointed out that the corresponding nursing measures can improve the patients' compliance, and mental symptoms [22].

\subsubsection{Epilepsy}

In the rTMS treatment, the incidence of epilepsy is very low, but seizures are still being considered the most serious risk [23]. Studies have shown that predisposing factors, stimulus intensity, frequency, stimulation site with patients are related to seizures [24]. Therefore, patients must be adequately assessed before the treatment, excluding history of seizures or strong positive family history of epilepsy, patients with severe somatic diseases, serious alcohol abusers, patients implanted heart pacemakers, children or pregnant women, history of brain surgery, with metal implants in the brain, and patients suffering from diseases of the nervous system [25]. In the rTMS treatment, we should closely observe patients with dizziness, headache, body twitch, etc. When patients occur twitches, immediately stop the treatment, assist the patient supine, put head to one side, move the removable dentures out, loosen the collar and waistband, place a spatula or mouth speculum between the upper and lower molars, timely liquidate the secretions of respiratory tract, if necessary, give negative pressure suction with adequate protection of limbs, do not press down firmly in order to avoid dislocations or fractures [26]. When seizures stopped, the nursing stall should immediately send the patient back to the ward, give oxygen and ECG monitoring, strictly monitor the blood oxygen saturation, heart rate and blood pressure changes.

\subsubsection{Head and neck discomfort}

Headache for rTMS treatment is the most common adverse reaction, and the incidence is $10 \%$ 30\% [27]. Its related physiological and inducing factors are unknown so far [28]. When we conduct the rTMS treatment, we should observe whether the patient has headache or neck discomfort, as well as the pain area, nature, duration, and so on. If the patient occurs uncomfortable symptoms, let the patient rest, maintain the light tender, keep the temperature and humidity appropriate, use the method of massage and local hot compress, or use a sedative (such as aspirin) when necessary.

\subsubsection{Hearing impairment, tinnitus}

Some scholars reported that some patients after the rTMS treatment, the safety of pure tone listening test will be happening temporary changes [29]. Loo [30] found that rTMS can bring patients with mild hearing loss, it may be associated with the high frequency sound. He suggested to wear a headset to avoid the adverse reaction. So patients could wear a headset and listen the soft music to reduce the discomfort caused by the noise.

\subsection{To strengthen social support}

Patients with mental disorder as a special kind of vulnerable groups, are more in need of the society, health care workers and family's understanding and support. Good social support, especially the family support could greatly improve the compliance of patients. When patients receive the treatment for rTMS, family members should be done well emotional work and are encouraged to their company with the patients. The families and friends of patients should be to appeal to take care of them, go to the related knowledge lectures of treatment together and encourage patients to attend treatment. Good social support can not only improve the patient's symptoms, but can improve the patient's psychological state, make the patients face the treatment with a positive and optimistic attitude, so as to accelerate the recovery of disease. So the medical staff should strengthen the education of families to ensure they can provide better support and supervision to patients.

\section{Summary}

Mental illness is a chronic disease, which is easy to relapse. The demand of adherence to treatment is higher, therefore, it is significant to improve patients' compliance on disease treatment and control. In the process of treatment, nursing staffs should establish a good relationship with the patients, understand the various factors influencing the patients' compliance and adopt timely corresponding nursing measures. Currently, the sample of the studies on adherence of rTMS treatment of patients with mental disorders are small, and these studies not yet introduce the survey tool in detail ,the reliability and validity of the tool have not been tested. It will increase the sample size in the future research to get more comprehensive factors. The qualitative research should be taken into account, and the psychological factors in the patents and their families should be discussed in-depth in the further study. Comprehending the influence factors of patients' compliance from another perspective, then it will supply a better nursing care to patients, improve the treatment adherence and the effect of the care.

\section{Acknowledgment}

This work is supported by the Government Funding for Clinical Medicine Excellent Talents Training Project (No. 361014) and Medical Appropiate Technology Tracking Project of Hebei Province (No. G2015045). 


\section{References}

1. Chen $X H$, Ke $X Y$, Liu $X Y$, et al. Research on the specificity of nursing in schizophrenia patients with repetitive transcranial and magnetic stimulation. Chinese Journal of Practical Nursing. 2013;29(24):69-71.

2. Liu J, Yi ZH, Wang JJ. Research progress on treatment of cognitive dysfunction with repetitive transcranial and magnetic stimulation. Journal of Psychiatry. 2011;24(2):145-147.

3. Lehner A, Schecklmann M, Poeppl TB, et al. Efficacy and Safety of Repeated Courses of rTMS Treatment in Patients with Chronic Subjective Tinnitus. Biomed Res Int. 2015;2015(5):1-7.

4. Sun YH, He NT, Zhao K. A comparative study of repetitive transcranial magnetic stimulation with Theta burst stimulation paradigm to improve the symptoms and executive function in patients with schizophrenia. Journal of Psychiatry. 2016;29(1):26-28.

5. Wu TK, Chen XH, Lin L, et al. Research analysis on anxiety and compliance of the patients with repetitive transcranial and magnetic stimulation. Journal of Qilu Nursing. 2013;19(23):64-65.

6. Liu M. Research on anxiety and compliance of the patients with repetitive transcranial and magnetic stimulation. Public Health (Academic Edition). 2014;8(22):2.

7. Subramaniam M, Abdin E, Picco L, et al. Stigma towards people with mental disorders and its components - a perspective from multi-ethnic Singapore. Epidemiol Psychiatr Sci. 2016:1-12.

8. Li J, Li J. Research progress on stigma of mental disorders. Journal of Psychiatry. 2014;27(3):232-234.

9. Hovington $\mathrm{CL}$, Mcgirr A, Lepage $\mathrm{M}$, et al. Repetitive transcranial magnetic stimulation (rTMS) for treating major depression and schizophrenia: a systematic review of recent meta-analyses. Ann Med. 2013;45(4):308-321.

10. O'Reardon JP, Solvason HB, Janicak PG, et al. Efficacy and safety of transcranial magnetic stimulation in the acute treatment of major depression: a multisite randomized controlled trial. Biol Psychiatry. 2007;62(11):1208-1216.

11. Loo CK, Mcfarquhar TF, Mitchell PB. A review of the safety of repetitive transcranial magnetic stimulation as a clinical treatment for depression. Int J Neuropsychopharmacol. 2008;11(1):131-147.

12. Luo L. Research progress on treatment compliance and nursing intervention of diabetic patients. Journal of Youjiang Medical University for Nationalities. 2011;33(4):541-542.

13. Nie S, He HB, Zhu JY, et al. Effects of health education on illness knowledge of relatives of patients with mental disease and their attitude to patients. Chinese Clinical Nursing. 2015;7(4):340-342.

14. Wu DS, Dai L, Chen GW, et al. Economic burden of patients with severe mental disease and its influencing factors. Sichuan Mental Health. 2015;28(6):533-535.

15. Gong WL, Na LN, Luan Y. Research on psychological care of the patients with repetitive transcranial and magnetic stimulation. China Practical Medical. 2012;7(12):246247.

16. Gao XC. The treatment of psychological nursing intervention in 30 depression patients with repetitive transcranial and magnetic stimulation. Journal of Qilu Nursing. 2013;19(17):37-38.

17. Shen SY. Comparative study on repetitive transcranial and magnetic stimulation combined with psychological nursing treatment of generalized anxiety disorder. Chinese Journal of Convalescent Medicine. 2014;23(9):770-772.

18. Zheng W, Zhang HY. Clinical study on repetitive transcranial and magnetic stimulation combined with psychological nursing for the treatment of depression. Chinese Journal of Convalescent Medicine. 2013;22(12):1066-1067.

19. Ai CQ. The impact of comprehensive nursing intervention on treatment compliance of repetitive transcranial magnetic stimulation. Medical Journal of Chinese People's Health. 2012;24(8):924-925.

20. Bernard S, Westman G, Dutton P R, et al. A Psychiatric Nurse's Perspective: Helping Patients Undergo Repetitive Transcranial Magnetic Stimulation (rTMS) for Depression. J Am Psychiatr Nurses Assoc. 2009;15(5):325-332.

21. Chen XH, Ke XY, Liu XY, et al. Research on the specificity of nursing in schizophrenia patients with repetitive transcranial and magnetic stimulation. Chinese Journal of Practical Nursing. 2013;29(24):69-71.

22. Wang $\mathrm{YH}, \mathrm{Li} \mathrm{YL}$. Influence of nursing intervention on patients with schizophrenia receiving repetitive transcranial magnetic stimulation. Chinese Journal of Practical Nursing. 2015;31(33):2535-2537.

23. Anderson B, Mishory A, Nahas Z, et al. Tolerability and safety of high daily doses of repetitive transcranial magnetic stimulation in healthy young men. $J$ Ect. 2006;22(1):49-53.

24. Guo FL. Research on clinical application of repetitive transcranial magnetic stimulation in patients with vegetative state. Kunming Medical University. 2014.

25. Bakker N, Shahab S, Giacobbe P, et al. rTMS of the dorsomedial prefrontal cortex for major depression: safety, tolerability, effectiveness, and outcome predictors for 10 $\mathrm{Hz}$ versus intermittent theta-burst stimulation. Brain Stimul. 2015;8(2):208-215.

26. Shen AX, Qian ML, Gu Q, et al. Research on nursing in Generalized Anxiety Disorder with repetitive transcranial and magnetic stimulation. Nursing and Rehabilitation Journal. 2010;9(6):484-485.

27. Zhao JP. Schizophrenia. Beijing:People's Health Publishing House. 2012;376-377.

28. Coarkin PE, Wall CA, King JD, et al. Pain during transcranial 
magnetic stimulation in youth. Innov Clin Neurosci. 2011;8(12):18-23.

29. Pascual-Leone A, Houser CM, Reese K, et al. Safety of rapid-rate transcranial magnetic stimulation in normal volunteers. Electroencephalogr Clin Neurophysiol. 1993;89(2):120-130.
30. Loo C, Sachdev P, Elsayed $\mathrm{H}$, et al. Effects of a 2- to 4week course of repetitive transcranial magnetic stimulation (rTMS) on neuropsychologic functioning, electroencephalogram, and auditory threshold in depressed patients. Biol Psychiatry. 2001;49(7):615-623. 